\title{
UNA MIRADA AL PENSAMIENTO EDUCATIVO DE LUIS BELTRÁN PRIETO FIGUEROA
}

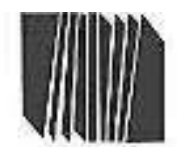

Unavosiabap proarsoaice

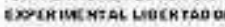

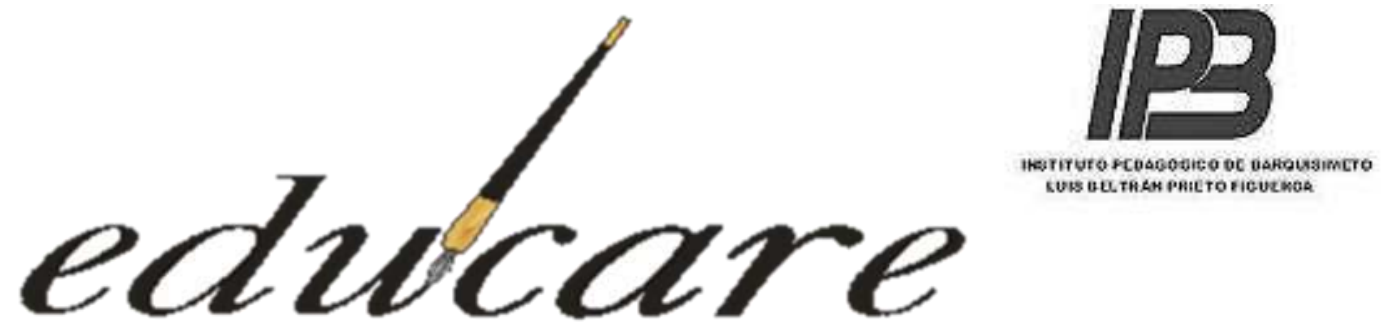

Órgano Divulgativo de la Subdirección de Investigación y Postgrado del Instituto Pedagógico de Barquisimeto "Lıis Beltrán Prieto

Figueroa"

\section{Volumen 22 № 3}

Septiembre - Diciembre 2018

UNA MIRADA AL PENSAMIENTO EDUCATIVO DE LUIS BELTRÁN PRIETO FIGUEROA

A LOOK AT THE EDUCATIONAL THINKING OF LUIS BELTRÁN PRIETO FIGUEROA

\section{S S N : $2244-7296$}

\author{
Autor \\ Alirio José Abreu Suarez \\ Ministerio del Poder Popular Para la Educación (MPPE) \\ Venezuela
}




\section{UNA MIRADA AL PENSAMIENTO EDUCATIVO DE LUIS BELTRÁN PRIETO FIGUEROA}

Alirio José Abreu Suarez

Ensayo

Recibido:05-03-2018

\section{RESUMEN}

La vida y obra del maestro Luis Beltrán Prieto Figueroa, es un referente para todos los actores del proceso educativo de los venezolanos. Prieto el político, maestro, estadista y gremialista son algunas razones para dedicarle tiempo a su pensamiento e ideas educativas. En ese sentido, se realiza en este ensayo una reflexión acerca de su percepción del tema educativo como: la escuela activa, escuelas rurales y Estado docente, en él se utilizan fuentes bibliográficas documentales. La obra de Prieto está al servicio de los más débiles muestra de eso fue del criterio de una educación de masas y no de castas. Por lo expuesto, se puede mencionar lo interesante que es para los educadores venezolanos conocer la obra de Prieto Figueroa, como una referencia existencial humana. Finalmente, se busca proporcionar información la cual pueda ser utilizada en diferentes trabajos e investigaciones por parte de los involucrados a la actividad educativa.

Palabras claves: Estado docente, educación, escuela activa.
Alirio José Abreu Suarez*
$M P P E$

Aceptado:15-09-2018

\section{ABSTRACT}

The life and work of Master Luis Beltrán Prieto Figueroa, is a benchmark for all actors in the education process of Venezuelans. Prieto the politician, teacher, statesman and unionist are some reasons to devote time to his thinking and educational ideas. In this sense, a reflection is made in this essay about his perception of the educational subject such as: The Active school, rural schools and the teaching State, it uses documentary bibliographical sources. Prieto's work is at the service of the weakest example of that was the criterion of a mass education rather than caste. For the above mentioned, we can mention how interesting it is for Venezuelan educators to know the work of Prieto Figueroa, as a human existential reference. Finally, it seeks to provide information which can be used in different jobs and research by those involved in the educational activity.

Key words: Teaching state, education, active school.

* LCDO en educación mención Ciencias Sociales UNESR. Especialista en planificación y evaluación educativa universidad Valle del Momboy ( UVM), especialista en educación para la recreación comunitaria, doctorante en la UNERMB. Docente activo en el MPPE 
UNA MIRADA AL PENSAMIENTO EDUCATIVO DE LUIS BELTRÁN PRIETO FIGUEROA

Alirio José Abreu Suarez

Pp 140-154

\section{INTRODUCCIÓN}

En el Siglo XX la sociedad venezolana fue testigo de los aportes al contexto educativo del maestro Luis Beltrán Prieto Figueroa, nacido el 14 de marzo del año 1902 en La Asunción Estado Nueva Esparta y fallecido en Caracas el 22 de abril de 1993. Sin duda que por su aporte a la educación pasa a ser considerado como uno de los insignes maestros Latinoamericanos del siglo pasado; sus ideas educativas aún son discutidas, debatidas y aceptadas por un gran número de docentes. Se le recuerda por ser un extraordinario político, escritor y crítico al sistema educativo imperante. Así como también defensor del Estado docente, es decir parte del principio de que tanto la educación pública y privada debe ser supervisada, orientada y ha de ser obligatoria para toda la sociedad.

En el mismo orden de ideas, fue un estudioso de educadores de reconocida talla como John Dewey, María de Pestalozzi, Adolphe Ferriere entre otros. Al respecto Luque (2010) señala: "Prieto Figueroa propuso la escuela activa que era, según él, algo más que un método de enseñanza" (p. 46) En otras palabras, buscó la transformación de la educación partiendo de erradicar la escuela tradicional, proponiendo el concepto de escuela nueva basado en el respeto e intereses de los educandos, utilizando metodología activa en donde se promueva la crítica.

En ese sentido, el sistema educativo actual toma en cuenta las ideas educativas de Prieto Figueroa. El Estado docente planteado por el maestro Prieto está plasmado en la ley orgánica de educación del año 2009 en los artículos 5 y 6. En esa ley el Estado asume el proceso educativo como un proceso esencial que promueve y difunde los valores y la dirección de la educación por parte del Estado en escuelas, liceos, universidades y demás instituciones escolares. Una de las razones por las que se le dan credibilidad a sus ideas educativas es que desde muy joven tuvo la responsabilidad de ser docente de escuela, presenciar, y describir cómo funcionaba la estructuración del sistema educativo venezolano.

Por otro lado, fue el fundador de la Sociedad Venezolana de Maestros de Instrucción Primaria el 15 de enero de 1932 ( SVMIP). A esa organización más tarde se le denominaría 
UNA MIRADA AL PENSAMIENTO EDUCATIVO DE LUIS BELTRÁN PRIETO FIGUEROA

Alirio José Abreu Suarez

Pp 140-154

como: Federación Venezolana de Maestros (FVM) en 1936, gremio que fue finalmente reconocido por el gobierno de Eleazar López Contreras. Institución fundada con el apoyo de otros notables como Luis Padrino, Mercedes Fermín, entre otros grandes educadores. Ellos lograron cumplir con ese objetivo y emprendieron una constante lucha en hacer propuestas para mejorar las reivindicaciones magisteriales en el aspecto económico, social y académico de los docentes.

Es de resaltar, que para ese tiempo no era fácil conformar y legalizar una organización sindical ya que esos hombres y mujeres estuvieron en constante vigilancia por los organismos de seguridad del Estado, que para el entonces eran manejados por el dictador Juan Vicente Gómez persona caracterizada por perseguir maestros, estudiantes, políticos o todo aquel que tuviera ideas progresistas en cualquier disciplina de las ciencias sociales.

Sumado a lo expuesto, fue corredactor de propuestas de leyes educativas para ser presentadas al Congreso Nacional del entonces, así como también un carismático líder gremial, político y estadista. Siguiendo a Luque (ob.cit) indica: "Desde 1936, ese grupo de héroes culturales tuvo una importante responsabilidad en la conformación de importantes organizaciones de la sociedad civil: partidos políticos sindicatos, agrupaciones culturales. Su acción creadora se extendió a toda la sociedad”. Es decir, las propuestas educativas de Prieto Figueroa tomaron gran fuerza en el territorio nacional. También fundó con los demás líderes gremiales La Revista Pedagógica como órgano informativo en donde se plasmaban experiencias educativas, contribuyendo de esa manera a mantener la dignidad docente como respuesta a una época de ignorancia, represión y poca información respecto a temas relacionados con la educación.

De acuerdo con Prieto Figueroa, la educación en Venezuela necesitaba de un giro en sus políticas educativas ya que la impartida en el siglo XX no respondía a las exigencias del país de aquel entonces. En ese sentido, Prieto (1978) manifiesta: "Siempre he sostenido que la educación no se puede medir por el volumen de los gastos ni por el presupuesto que se invierta en ella" 
UNA MIRADA AL PENSAMIENTO EDUCATIVO DE LUIS BELTRÁN PRIETO FIGUEROA

Alirio José Abreu Suarez

Pp 140-154

(p.144). Interpretando al autor, la educación debe de medirse por la eficiencia del sistema y por la calidad de este.

\section{LA ESCUELA ACTIVA DESDE EL PENSAMIENTO EDUCATIVO DE LUIS BELTRÁN PRIETO FIGUEROA}

La escuela activa en Venezuela es un modelo de institución escolar que surge en este país a raíz de la llamada escuela nueva nacida en Europa de la mano de grandes pedagogos como Ferrer Guardia y Giner de los Ríos en España, Adolphe Ferriere en Suiza, John Dewey en Estados Unidos, Lorenzo Miliani en Italia, entre otros ejemplares educadores. Todos ellos se basaron y sustentaron sus ideas educativas en maestros como María Montessori, Frederick Frobel, Ovidio Decroly y Edward Claparede por nombrar algunos. La escuela activa significa nueva educación o una manera distinta de enfocar la misma. En otras palabras, fue un movimiento que se desarrolló en los últimas décadas del siglo XIX como fuerte respuesta en oposición a la pedagogía tradicional buscando el valor humano, la dignidad y erradicando el autoritarismo.

Por su parte, en los países Iberoamericanos a esa escuela se le conoce como escuela activa surgida de la mano de uno de los tantos hombres que han contribuido con mejorar la educación en la región como lo es Tomás Vasconcelos. Al respecto Ocampo (2005) dice: "Este intelectual cuando ejerció la secretaría de instrucción pública en México, ejerció una de las más grandes reformas educativas y culturales en México e Iberoamérica en la primera mitad del siglo XX, que es ejemplo para Latinoamérica y el mundo" (p.2). Por lo antes expuesto, se considera el aporte de Vasconcelos como primordial para la escuela activa en la región por ser además un humanista, estadista e ideólogo. Uno de sus planteamientos fue construir una educación para las nuevas generaciones Por otro lado, en referencia a la escuela activa en Iberoamérica Narváez (2006) manifiesta:

En determinados países -en Iberoamérica, por ejemplo- al movimiento de la Escuela Nueva se le llama Escuela Activa, dado que se asume que el principio más importante y significativo es aquel según el cual la escuela debe propiciar la 
UNA MIRADA AL PENSAMIENTO EDUCATIVO DE LUIS BELTRÁN PRIETO FIGUEROA

Alirio José Abreu Suarez

Pp 140-154

actividad del niño; de allí que se sostenga que esta institución está llamada a aplicar el principio de actividad y hacer posible toda la actividad psicomotora propia del niño, a fin de centrarse en los intereses de éste y hacer más eficaces la enseñanza y el aprendizaje ( p.1).

En efecto, la escuela activa o también nueva es un espacio para el respeto a las distintas ideas, pensamientos e idiosincrasia. Igualmente se caracteriza por la compañía que los diversos actores del proceso educativo puedan ofrecer a los estudiantes siendo cuidadosos con el avance de los educandos. También la escuela entra en acción en beneficio de los niños fomentando responsabilidades en proceso de formación de los estudiantes.

De la misma manera, Mogollón (2011) señala: "La Escuela Activa es un enfoque pedagógico integral que promueve la instrucción personalizada y la creación de vínculos fuertes entre la escuela y la comunidad para asegurar que los niños y niñas aprendan competencias que les sirvan para la vida" (p.4). De acuerdo al autor, la educación ha de estar al servicio de los seres humanos para que estos sirvan al prójimo y al mismo tiempo aprovechar los lazos que puedan existir con otros actores como las fuerzas vivas de la sociedad para que contribuyan al proceso educativo de los educandos.

Del mismo modo, Menin (1998) expresa: "Lo que se ha denominado «escuela nueva» aparece en América Latina con su impronta renovadora bajo diversos registros, tanto políticos como epistemológicos" (p.1). Según el autor, los cambios que se puedan efectuar en una sociedad sean tanto en el mundo de la política, la economía y el avance de la ciencia guarda relación con los sistemas educativos. En otras palabras, tanto la escuela activa y la nueva cumplen las mismas funciones como lo son: incorporar a los diversos actores del proceso educativo; la formación docente, formar ciudadanos críticos y participativos; fomentar la cooperación y trabajo en equipo en otros aspectos. Es de destacar que la escuela nueva Latinoamericana guarda estrecha relación con la escuela activa europea ya sé que puede decir que es en gran parte una réplica de ésta.

Asimismo, en Venezuela Luis Beltrán Prieto Figueroa plantea una escuela activa que esté al servicio de los más necesitados es partidario de masificar la educación sin que se sacrifique la 
UNA MIRADA AL PENSAMIENTO EDUCATIVO DE LUIS BELTRÁN PRIETO FIGUEROA

Alirio José Abreu Suarez

Pp 140-154

calidad educativa en donde las fortalezas de los educandos sean tomadas en cuenta con el fin de aprovecharlas. También dice que las escuelas deben fomentar el espíritu de la participación, cooperación y la solidaridad además de ser espacios para la investigación y el deporte algo que no se estaba dando en el sistema educativo.

En ese sentido, es pertinente resaltar que el maestro Prieto Figueroa estaba consciente de lo importante que era promover la investigación en las instituciones escolares como punto de partida para desarrollo de una Nación. Aunado a lo expuesto, planteaba una escuela en donde se compartieran experiencias educativas, se promoviera el respeto, la ayuda mutua. Es otras palabras, una institución al servicio de la gente.

Para Prieto la escuela nueva debe tratar al niño tal como es y no considerarlo un adulto ya que él piensa, actúa, siente y padece. Coincidiendo en ese sentido con Jean Jacques Rousseau, el cual se oponía a doctrinas escolásticas. De igual manera, partió del principio de que el estudiante debe contribuir con su aprendizaje, es decir enseñarse a sí mismo, que se vuelva educador de sí mismo y se convierta en autodidacta. A la vez, planteaba que en las instituciones educativas se ha de considerar el humanismo democrático como estilo de participación social.

En el prólogo del maestro como líder Londoño (citado en Prieto, 2012) lo define:

El humanismo democrático consiste en la educación integral y se logra por la adquisición de conocimientos técnicos para la explotación utilización de los bienes con la aplicación de los fines utilitarios de la vida; de modo análogo, selecciona los conocimientos culturales, propiamente formativos en la democracia y de la personalidad : designa la necesidad de capacitar a los ciudadanos para la convivencia en las instituciones libres; en consecuencia, se requiere educar para el aprecio y la defensa de la libertad, la igualdad, la solidaridad y la responsabilidad, que son los ejes de la vida democrática ( p.47)

Interpretando al autor, la escuela ha de formar personas con un alto sentido de sensibilidad social en donde todo el saber esté al servicio del colectivo. La educación que reciba el niño debe ajustarse a las necesidades del país, lo que es igual a formar personas que impulsen el aparato productivo en áreas como la agricultura, la ganadería, hidrocarburos o cualquier otro, estos deben ser formados para que tengan conciencia con la responsabilidad que tienen en la sociedad. De 
UNA MIRADA AL PENSAMIENTO EDUCATIVO DE LUIS BELTRÁN PRIETO FIGUEROA

Alirio José Abreu Suarez

Pp 140-154

igual manera, a los educandos se les ha de manifestar lo importante que es la democracia como instrumento de vida de los ciudadanos. Lo expuesto es posible desde la escuela activa planteada por Prieto, ya que ésta pretende formar individuos aptos para la crítica, autocritica y con capacidad de ser parte de la soluciones.

De la misma manera, Prieto consideró el papel protagónico del maestro en la escuela nueva y la comunidad considerándolo un líder por ser una persona cercana a: los estudiantes, representantes y fuerzas vivas de la sociedad. Por lo expuesto, el docente según la visión del maestro Prieto Figueroa, es capaz de solventar diversas situaciones que se puedan presentar. Sumado a lo planteado, es capaz de organizar y organizarse para eventos que se puedan presentar ya que está formado para eso y por tener una alta sensibilidad social. En la escuela planteada por Prieto, el docente debe hacer diversos trabajos tanto teóricos y prácticos desde la agricultura, carpintería, albañilería, herrería, principios básicos de farmacia, medicina y demás. Eso con el fin de enseñar a los estudiantes un oficio que le pueda servir en el futuro al respecto Prieto (2012) indica:

El Maestro llego a las comunidades con su nombramiento debajo del brazo, algunas veces sin conocimientos del medio rural, pero animado de un sano idealismo de servicio, y se entregó a la tarea de hacer. Se le pedía que diese cada día más, y en algunos casos con los vecinos, otras solo o con sus alumnos, construyó escuelas, organizó huertas experimentales, construyó muebles, fundó el periódico rural y el teatro popular, y se hizo manualista, médico y boticario, todo en una sola pieza (p.95).

Ante lo expuesto, para el líder magisterial, el ser docente significa estar de acuerdo con aquellos cambios que ayuden a mejorar el entorno escolar. La escuela tiene el papel de formar hombres críticos, pero también personas que aprendan oficios que le sirvan en la vida, planteamiento en el cual coincidía con otro gran maestro como lo fue Simón Rodríguez. Igualmente (ob.cit:) señala. "La escuela y los maestros tienen tareas que no pueden eludir" En ese sentido, la escuela activa ha de aprovechar todas las fortalezas de los directivos, docentes, representantes, estudiantes y comunidad en general para optimizar la educación y a la vez solventar cualquier situación que se presente. La escuela ha de ser un ente activo entre su personal y la comunidad. Por ser el maestro una persona con un conocimiento mayor al de estos ha de ser el guía o líder 
UNA MIRADA AL PENSAMIENTO EDUCATIVO DE LUIS BELTRÁN PRIETO FIGUEROA

Alirio José Abreu Suarez

Pp 140-154

\section{LA ESCUELA RURAL DESDE LAS IDEAS EDUCATIVAS DE PRIETO FIGUEROA.}

Luis Beltrán Prieto Figueroa, un educador de una alta sensibilidad social trabajo' en varios proyectos con el fin de mejorar la educación Venezolana. Así mismo, se preocupó por el funcionamiento de las escuelas rurales, las cuales surgen a raíz de la fundación de la escuela normal rural el Macaro, como centro especializado de formación, institución fundada en el gobierno de Eleazar López Contreras producto de la misión de los pedagogos Chilenos. Esa misión chilena tuvo como objetivo primordial brindar asesoría técnica, pedagógica, elaboración de planes y programas dirigidos al ministerio de educación y al magisterio venezolano.

Sin embargo, Luis Padrino le dio la dirección correcta a esa institución en su paso por el Ministerio de educación, pero Prieto en sus escritos y reuniones aportó algunas ideas así lo reconoce Padrino (citado en Luque, 2013) "Se debe a la labor tesonera, persistente y tenaz de la Federación Venezolana de Maestros en pro del niño, la escuela y la cultura patria y, en definitiva, a la persistente labor del Dr. Luis Beltrán Prieto Figueroa” (p.69). Al respecto, se manifiesta el reconocimiento a la obra educativa en el área rural del maestro Prieto, por parte de la persona que se le atribuye la creación de las escuelas rurales en Venezuela. Al respecto, se manifiesta el reconocimiento a la obra educativa en el área rural del maestro Prieto. Como ministro de educación crea las escuelas normales rurales: Yocoima en el Estado Bolívar y Gervasio Rubio en el Táchira.

De igual manera, Prieto siempre se preocupó por la alta formación del docente en las escuelas rurales por considerar que su papel de trabajo se efectuaría con una alta masa de campesinos, agricultores, es decir personas sin recursos económicos dedicados a las áreas productivas del país, pero con un elevado índice de analfabetismo sin acceso a los servicios básicos como: salud, educación, transporte entre otros.

En el mismo orden de ideas, el maestro Prieto Figueroa fue partidario de la masificación de la educación y para eso consideró que eso es posible si se incorporan el mayor número de 
UNA MIRADA AL PENSAMIENTO EDUCATIVO DE LUIS BELTRÁN PRIETO FIGUEROA

Alirio José Abreu Suarez

Pp 140-154

escuelas rurales y se preparan docentes. Es de precisar que los maestros y en especial los de la zona rural tienen una enorme responsabilidad de la formación, autoformación y estudio voluntario con el propósito de dar lo mejor de sí a la escuela, comunidad, niños, niñas y adolescentes y a la sociedad en sí.

Asimismo, Luis Beltrán Prieto Figueroa estuvo consciente del estado de abandono del campesino y vio en México una posibilidad en cuanto a aplicar ese modelo educativo o funcionamiento de las escuelas rurales en Venezuela. Partió de la tesis de la formación del docente rural como instrumento de formación de las masas. Reconociendo éste la labor de labor de Luis Padrino al frente de esa institución y la importancia para la nación Prieto (citado en Luque 2013) señala:

Luis Padrino regresó a Venezuela después de dos años de asiduos estudios e investigaciones acuciosas de los métodos y procedimientos de la educación rural en México, donde viajó y trabajo' con el propósito de aquilatar conocimientos utilizables en Venezuela para una obra de elevación cultural de nuestra masa campesina, y de mejoramiento humano de nuestro pueblo (p.235)

Sumado a lo expuesto, sostuvo que el campesinado no importaba para los administradores de la educación. Consideró que los convenios internacionales en materia educativa es una necesidad para las naciones ya que en estos se adquieren experiencias educativas, compartir de saberes, adquisición de metodologías que se pueden aplicar en el país con el fin de mejorar la educación. Es decir, imitar lo positivo o experiencias que han sido satisfactorias para algunos países y trabajar en base a ellas. Las escuelas en esos tiempos estaban al servicio de una minoría lo que era igual a una educación de castas concepción defendida por algunos ideólogos del Gomecismo u otros notables escritores que pensaron distinto. Prieto fue un arduo defensor de la masificación de la educación, pero sin sacrificar la calidad educativa es así como sostuvo diferencias con grandes intelectuales como: José Gil Fortoul, Samuel Darío Maldonado y Felipe Guevara Rojas.

Con relación al planteamiento anterior el maestro Prieto Figueroa, sostuvo que la educación es un derecho para todo ciudadano sin importar su condición social, religión, 
UNA MIRADA AL PENSAMIENTO EDUCATIVO DE LUIS BELTRÁN PRIETO FIGUEROA

Alirio José Abreu Suarez

Pp 140-154

tendencia política, sexo o cualquier otro elemento presente en la vida de las personas. Además de considerar a la población rural como vulnerable, a la vez la consideró importante en el desarrollo del país, también partió del principio de educarlo y darle oportunidades. .

\section{EL ESTADO DOCENTE DESDE EL PENSAMIENTO EDUCATIVO DE LUIS BELTRÁN PRIETO FIGUEROA.}

Luis Beltrán Prieto Figueroa, al igual que otro grande de América como lo fue Paulo Freire, mantuvo la posición que la educación debe ser direccionada por un ente rector. Siempre defendió el papel del Estado en los asuntos relacionados con la educación, en otras palabras partió del criterio de que es el responsable de las políticas educativas. Éste implementa los programas educativos, orienta, realiza el control de todas las políticas en materia educativa a eso se define como Estado docente.

De igual manera, consideró al Estado como el ente ejecutor de la actividad educativa, Prieto (2006) indica: "En un país cualquiera, una época cualquiera es inconcebible que el Estado deje abandonada al capricho de las actividades particulares la orientación y formación de la conciencia de los ciudadanos" (p.27). En torno a la posición del autor, la educación en todos sus niveles y modalidades ha de ser orientada por el Estado; a esta no escapa la universitaria a la misma. Respecto a las universidades, Prieto (ob.cit:) dice: "No puede seguirse mintiendo sobre la independencia de la universidad respecto del Estado mientras sea instrumento de fuerzas internas o externas que la ponen al servicio de mezquinos y transitorios intereses”. Así reconoce el papel de la universidad en el desarrollo del País, a la vez exhorta a las autoridades a dejar las diferencias de distinta índole que se puedan tener ya que eso retrasa el desarrollo de la nación.

En el mismo orden de ideas, plantea que toda educación es ideológica respondiendo la misma a la naturaleza o ideología política del gobierno de turno. Ese planteamiento no es de extrañar a las personas, se debe considerar eso como una situación natural en donde no puede haber resistencia en los diversos sectores de la sociedad. Asimismo Freire (2004) manifiesta: 
UNA MIRADA AL PENSAMIENTO EDUCATIVO DE LUIS BELTRÁN PRIETO FIGUEROA

Alirio José Abreu Suarez

Pp 140-154

"La Conciencia activa y dinámica del Estado es un sistema de pensamiento, de ideas, de intereses que hay que satisfacer y de moralidad que hay que realizar" (p.29) Parafraseando al maestro brasilero, el Estado ideologiza a la sociedad a través de su educación, toda ideología es el sostén de los gobiernos de turno. De igual manera, Prieto (2006)

El Estado interviene, por derecho propio, en la organización de la educación del país, y orienta, según su doctrina política, esa educación. Depende la orientación de una escuela de la orientación política del Estado. Si el Estado es fascista, la escuela es fascista. Si el Estado es nazista, la escuela es nazista. Si el Estado es falangista, la escuela es falangista. Y si el Estado es democrático, la orientación de la escuela necesariamente tiene que ser democrática (p.27).

Igualmente, es pertinente resaltar que Prieto Figueroa, condenaba los gobiernos totalitarios como: el Fascismo de la Italia de Mussolini, Nazismo de Hitler y el Franquismo en España por ser estos violadores de los derechos humanos como: el derecho a la vida, la libre prensa y el funcionamientos de los partidos políticos entre otros aspectos. Sin embargo, no condenaba la ideologización que estos impartían a sus ciudadanos ya que defendía la tesis del rol del Estado en la educación.

La escuela es el lugar perfecto para dar inicio al proceso de ideologización de los hombres desde niños se les describen los beneficios o bondades de un sistema político u otro. Así como también, se les dice cuáles son los amigos o enemigos del país, a la vez se diseñan en los diferentes currículos que se debe impartir en las instituciones escolares. Todo eso busca el objetivo de crear adeptos al sistema imperante y eso se hace en cualquier sistema político partiendo desde la antigua Grecia, el imperio Romano, el sistema feudal, hoy en Corea del Norte o Sur, es decir siempre estará inmersa la orientación política e ideológica en la educación.

De igual manera, Prieto fue un estudioso de distintos sistemas educativos imperantes de Europa en vísperas de la segunda guerra mundial aunque no compartía su posición política e ideológica los analizó desde el punto de vista político ideológico y político. Entendió que el Estado se sustenta en un conjunto de acciones, pensamientos. Es de destacar que la ideología política de un Estado es transmitida e impartida en las instituciones escolares por los maestros, 
UNA MIRADA AL PENSAMIENTO EDUCATIVO DE LUIS BELTRÁN PRIETO FIGUEROA

Alirio José Abreu Suarez

Pp 140-154

visto de esa manera se convierte en docente activo e ideologizador de las diferentes generaciones de estudiantes.

Del mismo modo, Luis Beltrán Prieto Figueroa define la educación como una actividad que genera cambios positivos en los seres humanos, esta es organizada y sistematizada por el ente que le corresponde en este caso el Estado. En las sociedades la educación se convierte en un fenómeno de la colectividad y el Estado fija los instrumentos y normas para hacerla posible. Resulta claro, que en todo proceso educativo todos los actores juegan un papel importante en cuanto a la consecución de objetivos que favorezcan la calidad educativa. Para lograr eso es indispensable la intervención del Estado y que éste la asuma como una obligación para todas las sociedades.

\section{COMENTARIOS FINALES}

El aporte que el maestro Prieto, le dio a la educación venezolana es sin duda muy significativo como hombre formado en tiempos de dictadura, comprendió lo peligroso que es para una nación un gobierno de esa naturaleza. Además, de ser un luchador social, líder magisterial, docente de la escuela primaria, político y gran estadista vio en la educación la única vía o instrumento de desarrollo de la sociedad. De hecho, fundó La Revista Pedagógica como medio de información del magisterio en el cual se manifestaban experiencias educativas, propuestas y posibles soluciones a la grave problemática educativa de la nación. Además, se le considera junto a Luis Padrino, Mercedes Fermín, Belén de san Juan y otros los pioneros de la escuela activa venezolana.

En el mismo orden de ideas, defendió la función del Estado en el ejercicio docente lo califica como un ente orientador y ejecutor de la políticas educativas de los gobiernos de turno, aunque no compartió en lo político con los regímenes dictatoriales entendió las razones que llevan a estos a utilizar la educación como un mecanismo ideologizador de las masas.

En síntesis, las ideas educativas del maestro Prieto, siempre estarán presentes en el magisterio. En estos tiempos se le recuerda como: el líder gremial que fundó junto a otros la 


\section{UNA MIRADA AL PENSAMIENTO EDUCATIVO DE LUIS BELTRÁN PRIETO FIGUEROA}

Alirio José Abreu Suarez

Pp 140-154

FVM con el fin de dignificar a los docentes; defensor de la tesis del Estado docente; partidario de una educación de masas y no de élites; político y ex candidato presidencial; hombre de una alta sensibilidad social y muchos otros elementos positivos que se evidenciaron durante su trayectoria política y educativa al servicio de la sociedad. Del mismo modo, como se le recuerda a Simón Rodríguez por ser el maestro de más relevancia durante el siglo XIX, hoy el maestro Prieto se considera el docente venezolano de mayor trascendencia en el siglo XX.

\section{REFERENCIAS.}

Freire, (2004). Pedagogía de la autonomía. Sao Paulo. Editorial Paz e Terra SA

Luque, G (2010). Educación pueblo y ciudadanía. Caracas. Editorial el perro y la rana.

Luque, G (2013). Luis Padrino, maestro de la escuela nueva y fundador de la educación rural en Venezuela. Caracas. Editorial UCV.

Menin, O (1998). El ensayo de «escuela serena» realizado por las hermanas Cossettini en la República Argentina. Rev. Fac. Educ. vol.24 n.1 São Paulo Jan./Jun. 1998[Artículoenlínea].Disponible:http://www.scielo.br/scielo.php?script=sc i_arttext\&pid=S0102-25551998000100011[Consulta: Agosto, 03].

Mogollón, O (2011). Escuelas activas Apuestas para Mejorar la Calidad de la Educación. Revista escuelas activas. [Revista en línea].Disponible en http://www.epdc.org/sites/default/files/documents/Active_Schools_Spanish.pdf [Consulta: Agosto, 01]

Narváez, E (2006). Una mirada a la escuela nueva. Educere v.10 n. 35 [Artículo en línea].Disponible:http://www.scielo.org.ve/scielo.php?script=sci_arttext\&pid=S131649102006000400008[Consulta: Agosto, 02]

Ocampo, J (2005). José Vasconcelos y la Educación Mexicana Revista Historia de la Educación Latinoamericana, vol. 7, 2005. [Artículo en línea]. Disponible en http://www.redalyc.org/pdf/869/86900707.pdf [Consulta: Agosto, 01]

Prieto, L (1978). Conversaciones con Luis Beltrán Prieto Figueroa. Caracas. Editorial Ateneo de Caracas.

Prieto, L. (2006). El Estado docente. Caracas. Editorial Fundación Biblioteca Ayacucho. 
UNA MIRADA AL PENSAMIENTO EDUCATIVO DE LUIS BELTRÁN PRIETO FIGUEROA

Prieto, L. (2012).El maestro como líder. Caracas. Editorial Fundación Biblioteca Ayacucho. 